\title{
Competencia científica de estudiantes que ingresan y egresan de la Universidad
}

\section{Science competency of students enrolling and graduating at University}

\author{
C. B. Falicoff, H. S. Odetti \\ Departamento de Química General e Inorgánica, Facultad de Bioquímica y Ciencias Biológicas, UNL. \\ Ciudad Universitaria Paraje El Pozo. CC 242. (3000), Santa Fe, Argentina. \\ falicoff@fbcb.unl.edu.ar, hodetti@fbcb.unl.edu.ar \\ J. M. Domínguez Castiñeiras \\ Departamento de Didáctica das Ciencias Experimentais, Facultade de Ciencias da Educación, USC. \\ Avda. Xoan XXIII, s/n. 15704. Santiago de Compostela, Espańa. \\ josemanuel.dominguez@usc.es
}

RESUMEN • En el presente estudio se presentan resultados que ponen de manifiesto con qué competencia científica ingresan los estudiantes en la Facultad de Bioquímica y Ciencias Biológicas (primer curso de las carreras de Bioquímica y de Biotecnología de la Universidad Nacional del Litoral de Santa Fe, Argentina) y con cuál egresan de ella (quinto curso de las mismas carreras). Para obtener la información, se elaboraron dos cuestionarios ad hoc: uno con preguntas que abarcan las tres subcompetencias cientificas y otro para explorar la influencia de ciertas características del contexto sobre el rendimiento en estas.

Los resultados indican que los ingresantes parten de un nivel bajo en el rendimiento de todas las subcompetencias científicas evaluadas. Luego de la instrucción, en los últimos años, el desarrollo es favorable y de manera más apreciable en Bioquímica que en Biotecnología.

PALABRAS CLAVE: competencia científica; enseñanza universitaria; Química.

SUMMARY - This study presents results that show the science competency of students enrolling at the School of Biochemistry and Biological Sciences (first year of the courses of Biochemistry and Biotechnology of the Universidad Nacional del Litoral de Santa Fe, Argentina) and what competency they have when they finish University (fifth year of the same courses). To collect the information, two ad hoc questionnaires were drafted: one with questions addressing three science subcompetencies, the other one was used to examine the influence that certain contextual factors might have had on these competencies performance.

The results indicate that first-year students start with a low performance level for all the science subcompetencies assessed. After instruction, in the last years, the competencies develop favorably, more so among students of Biochemistry than among those of Biotechnology.

KEYWORDS: science competency; higher education; Chemistry.

Fecha de recepción: septiembre 2012 • Aceptado: mayo 2013

Falicoff, C. B.; Domínguez Castiñeiras, J. M.; Odetti, H. S. (2014) Competencia científica de estudiantes que ingresan y egresan de la Universidad. Enseñanza de las Ciencias, 


\section{MARCO TEÓRICO Y FUNDAMENTACIÓN}

En este apartado, fundamentado en la literatura publicada sobre el tema, se pone de relieve la relevancia de la presente investigación desde dos perspectivas: las competencias básicas y la competencia cientifica que debe desarrollar el alumnado para una preparación más eficaz, desde el punto de vista de las carreras que van a estudiar y del estudio PISA 2006. Ambos puntos de vista sustentan el interés de este trabajo, que es abordar la relación entre la enseñanza y el desarrollo de competencias, en concreto, de la competencia cientifica, en la formación profesional que propone el ámbito educativo universitario en dos carreras cuya disciplina fundamental es la Química.

Un nuevo término ha aparecido recientemente con fuerza en el mundo educativo: competencia. La necesidad de conceptualizar el término competencia ha provocado la aparición de definiciones diversas y la revisión de algunas de ellas. A este respecto Cañas, Martín-Díaz y Nieda (2007) afirman que, aunque provenga del mundo laboral, en el ámbito educativo incluye capacidades relacionadas con el desarrollo personal y social de los alumnos que pueden servir para una educación de mayor calidad, equidad e incidencia en la práctica docente real en las aulas.

Las finalidades de las competencias básicas según Perrenoud (2004), De Pro Bueno (2007) y Zabala y Arnau (2007) son: integrar los aprendizajes, ponerlos en relación con distintos tipos de contenido, utilizarlos de manera efectiva y orientar la enseñanza (identificar contenidos y criterios de evaluación). Monereo y Pozo $(2001,2007)$ opinan que, desde la perspectiva de las competencias básicas, se pone énfasis en el aprendiz y afirman que hay motivos para suponer que las competencias no están previamente en los alumnos, sino que, desde una perspectiva vygotskiana, son construcciones sociales que deben ser internalizadas a través de la educación.

Por lo tanto, la Escuela, como la Universidad, deberían dotar a los estudiantes de las competencias necesarias para acceder a las culturas simbólicas que caracterizan a nuestra sociedad. Enseñar por y para las competencias puede ayudar a definir mejor las metas y los propósitos de la acción educativa.

Marco Stiefel (2008) argumenta que los autores que han profundizado en el tema de las competencias afirman que el proceso de su construcción tiene dos pasos que no tienen por qué ser simultáneos, pero sí estar interrelacionados: uno es el de la movilización de las fuentes y recursos cognitivos, y otro el de la transferencia de los aprendizajes. Dicha autora considera que las capacidades movilizan saberes limitados, normalmente de tipo procedimental, y que las competencias, por el contrario, se apoyan en saberes amplios y explícitos, entre los que se incluyen posibilidades de abstracción, generalización y transferencia. A este respecto, Zabala y Arnau (2007) señalan que enseñar y/o desarrollar competencias implica utilizar formas de enseñanza consistentes en dar respuestas a situaciones cercanas a la vida real, en un complejo proceso de construcción personal con ejercicios de progresiva dificultad y ayudas, según las características idiosincrásicas del alumnado.

A este respecto, Jiménez Aleixandre (2000) pone de manifiesto que los objetivos para la enseñanza de las ciencias están tanto relacionados con saber ciencias como con hacer ciencias. Desde este punto de vista, dicha autora señala la especial importancia de la resolución de problemas. En este mismo sentido se manifiestan Cañas, Martín-Díaz y Nieda (2007), que consideran necesario para el desarrollo de la competencia cientifica el uso de varias estrategias que compaginen presentaciones expositivas del profesor con propuestas de actividades variadas a los alumnos.

Desde esta perspectiva, hemos de poner de manifiesto que los currículos de las universidades argentinas están organizados por disciplinas científicas y los cursos que se ofertan enfatizan fuertemente el conocimiento disciplinar. Es por tanto muy difícil imaginar a las universidades como las proveedoras de soluciones que pudieran ayudar al profesorado a mejorar la eficacia de sus prácticas docentes y a conocer las estrategias de aprendizaje de sus alumnos (Labate, 2007). Como señala Biggs (2005), a los profesores universitarios no les faltan teorías relacionadas con el contenido de su disciplina (aunque es- 
tas sean provisionales y/o controvertidas), sino teorías bien estructuradas relacionadas con la enseñanza de esta. Es necesario, por lo tanto, establecer espacios en los que puedan reflexionar para adquirir una teoría explícita de la enseńanza que les permita ver lo que está mal y cómo pueden mejorar su práctica docente. Esa práctica reflexiva e innovadora es la base de la profesionalidad para llegar a ser un mejor profesor (Shön, 1992).

Izquierdo Aymerich (2004) presenta dos posibles causas en la crisis de la enseñanza de la Química. En primer lugar, su presentación de manera demasiado dogmática, definiendo entidades que solo tienen sentido para los químicos y no planteando situaciones en las cuales la explicación química resulte relevante. En segundo lugar que, quizá, no se tienen en cuenta las dificultades conceptuales que se derivan del desajuste entre la teoría y sus ejemplos, modelos o campos de aplicación.

Si se centra la atención en la competencia científica, Chamizo e Izquierdo (2007) la entienden como el conjunto de capacidades que permiten saber, saber hacer, ser y vivir, con otros, en situaciones de la vida en las cuales se ha de decidir cómo actuar. Asimismo, Jiménez Aleixandre (2010) destaca aspectos como la capacidad de relacionar datos y conclusiones y de evaluar enunciados teóricos a la luz de los datos empíricos o procedentes de otras fuentes.

PISA tiene como objetivo generar indicadores comparativos internacionales de rendimiento educativo en los países miembros (OCDE, n.d.; Domenech, 2003). En este estudio se señala que la competencia científica requiere la comprensión de conceptos científicos y la capacidad para aplicar un punto de vista científico y pensar sobre las pruebas de una manera científica. Sus características distintivas son (OCDE, 2008, p. 23):

El grado en que un individuo:

- Tiene conocimiento científico y lo utiliza para identificar cuestiones, adquirir nuevos conocimientos, explicar fenómenos científicos y extraer conclusiones basándose en pruebas acerca de problemas relacionados con las ciencias.

- Comprende las características de la ciencia como forma de conocimiento e investigación.

- Es consciente de que la ciencia y la tecnología conforman nuestro medio material, intelectual y cultural.

- Se compromete como ciudadano reflexivo en problemas e ideas relacionados con las ciencias.

Las tres subcompetencias implicadas en la definición anterior, tal como se aplicaron en las evaluaciones de los años 2006 y 2009, se fundamentaron en la lógica, el razonamiento y el análisis crítico. Lo que sigue es una explicación más detallada de estas (OCDE, 2006; OECD, 2009).

\section{Identificar cuestiones científicas (ICC)}

Lo esencial en este caso es distinguir entre las cuestiones y contenidos científicos y otros tipos de cuestiones, como son:

- Reconocer cuestiones susceptibles de ser investigadas científicamente.

- Identificar términos clave para la búsqueda de información científica.

- Reconocer los rasgos clave de la investigación científica.

\section{Explicar fenómenos científicamente (EFC)}

Respecto a este segundo aspecto referido a las capacidades, se diferencia:

- Explicar fenómenos científicos.

- Aplicar el conocimiento de la ciencia a una situación determinada. 
- Describir o interpretar fenómenos científicamente y predecir cambios.

- Identificar las descripciones, explicaciones y predicciones apropiadas.

Los alumnos acreditan la capacidad de explicar sucesos científicamente.

\section{Utilizar pruebas científicas (UPC)}

Respecto a esta tercera subcompetencia, se tiene en cuenta:

- Interpretar pruebas científicas y elaborar y comunicar conclusiones.

- Identificar los supuestos, las pruebas y los razonamientos que subyacen a las conclusiones.

- Reflexionar sobre las implicaciones sociales de los avances científicos y tecnológicos.

Las declaraciones referidas a PISA ponen de manifiesto un giro en el enfoque de la enseñanza que recorre todas las etapas educativas, no solo las básicas, pues la renovación de la enseñanza universitaria propiciada por la creación del Espacio Europeo de la Educación Superior, a partir de Bolonia, va en la misma línea.

En este trabajo de investigación, como señalan Monereo et al. (2009) en el suyo, los estudios PISA son la excusa. Estos actúan como estímulo o inspiración para indagar las fortalezas y debilidades en cuanto a la competencia cientifica se refiere. Así, con los datos que emerjan de la investigación, poder reflexionar de qué manera se podrían favorecer los cambios positivos en las estrategias de aprendizaje de los alumnos y en las prácticas de enseñanza de los profesores.

Argentina ha participado como país asociado en 2000 y 2006, y los resultados de alumnos de 15 ańos (DINIECE-MEN, n.d.) ponen de manifiesto una diferencia estadísticamente muy significativa por debajo del promedio de la OCDE. Los alarmantes resultados, respecto a la adquisición de la competencia cientifica, llevan a varios interrogantes, por ejemplo: ¿cuáles son las causas de estos?, ¿qué ocurre en los niveles anteriores? Asimismo, en un nivel tan poco atendido como es el universitario, se considera relevante averiguar si estos pobres resultados permanecen en los estudios superiores.

La literatura sobre el tema pone de manifiesto que la perspectiva de enseñar por y para las competencias puede ayudarnos a definir las metas y los propósitos de la acción educativa. Ser competente no es solo ser hábil en la ejecución de tareas y actividades concretas, escolares o no, tal como han sido enseñadas, sino, más allá de ello, ser capaz de afrontar, a partir de las habilidades adquiridas, nuevas tareas o retos que supongan ir más allá de lo ya aprendido.

La intención de la presente investigación es dar un primer paso para diagnosticar algunos factores (De Pro Bueno, 2007) que pueden estar obstaculizando el incremento de la calidad de los procesos de enseńanza y aprendizaje en la Universidad argentina: la necesidad que tiene el profesorado de una formación adecuada a los nuevos tiempos y de disponer de materiales concretos para llevar a cabo los cambios en el qué y cómo enseñar en la práctica educativa; las decisiones administrativas en cuanto a programas oficiales, formación docente, recursos, etc., y las condiciones de acceso a la Universidad, ya que estas condicionan muchas actuaciones en los niveles no universitarios.

\section{Problemas de investigación}

De lo indicado hasta aquí se deriva la importancia que ha adquirido la competencia científica y el contexto de aprendizaje en los nuevos currículos. De este modo surge el interés en constatar en qué medida han desarrollado dicha competencia los estudiantes que ingresan en la Facultad y egresan de ella para su inserción en la vida profesional. Los resultados que se obtengan en la presente investigación deberían hacer posible la emisión de juicios de valor encaminados a determinar algunas de las características de los currículos universitarios, en las asignaturas de Química, que se podrían orientar sobre las 
prioridades para una enseñanza de calidad que fomente el desarrollo de la competencia investigada y algunas influencias del contexto sociocultural de los alumnos en su rendimiento en ciencias.

Teniendo en consideración lo anterior, se formulan los siguientes problemas de investigación:

- ¿Cuál es el grado de competencia científica que poseen los alumnos de primero y último año de las carreras de Bioquímica y Biotecnología?

- ¿Cuáles son las similitudes y diferencias entre la competencia científica de los estudiantes de primer y quinto año en ambas orientaciones universitarias?

- ¿Ciertas características del contexto sociocultural de los alumnos influyen en el rendimiento de la competencia científica?

Los estudios para obtener los títulos de bioquímico y biotecnólogo que otorga la Facultad de Bioquímica y Ciencias Biológicas de la Universidad Nacional del Litoral-Argentina (Validez Nacional por Resolución N. ${ }^{\circ}$ 1.567/1980 y Res. N. ${ }^{\circ}$ 1739/1997, respectivamente, del Ministerio de Cultura y Educación) tienen una duración de cinco años. A partir de los documentos oficiales de los currículos, para la identificación de los problemas de investigación se estimó conveniente explorar: los planes de estudios, el Sistema de Correlatividades, el Régimen Único de Enseñanza de la Facultad de Bioquímica y Ciencias Biológicas/Escuela Superior de Sanidad de la Universidad Nacional del Litoral y los perfiles de los títulos, tanto de las carreras de Bioquímica como de la de Biotecnología. Esta información está disponible en las siguientes páginas web: <http://www.fbcb.unl.edu.ar/pages/estudios/ carreras-de-grado/bioquimica.php $>$ y <http://www.fbcb.unl.edu.ar/pages/estudios/carreras-de-grado/ lic.-en-biotecnologia.php>.

\section{METODOLOGÍA}

\section{Sujetos y características de la muestra}

La muestra estudiada estuvo compuesta por 122 alumnos: 63 de Bioquímica y 59 de Biotecnología. El alumnado participante lo hizo de forma voluntaria.

El número de alumnos de cada submuestra se considera adecuado -mayor al 10\% de los inscritos, según la recomendación de Colás Bravo y Buendía Eisman (1998)-, y su composición es la siguiente:

- 47 estudiantes de primer año de Bioquímica (B), de un total de 110 inscritos, según las actas de alumnado de la Facultad de Bioquímica y Ciencias Biológicas (FBCB) N.o 2.275 y N.o 2.276 de 2010.

- 16 estudiantes del quinto año de Bioquímica (B), de un total de 27 inscritos, según las actas de alumnado de la FBCB N.o 2.224 y N.o 2.225 de 2010.

- 37 estudiantes de primer ańo de Biotecnología (LB), de un total de 73 inscritos, según el acta de alumnado de la FBCB N.o 2.215 de 2010.

- 22 estudiantes del quinto año de Biotecnología (LB), de un total de 45 inscritos, según el acta de alumnado de la FBCB N.o 2.249 de 2010.

Los instrumentos de recogida de información - dos cuestionarios- se les presentaron durante la segunda semana del año académico 2010 y no hubo adiestramiento previo del alumnado participante en las cuestiones inherentes a estos.

Para poder llevar a cabo la presente investigación, se considera necesario suponer qué conocimiento tienen los alumnos, en función de los estudios realizados: 
- Los alumnos de primer año han finalizado la fase de escolaridad obligatoria y han aprobado el ingreso en la Universidad. Se supone que han adquirido un conocimiento básico de Química, así como las destrezas básicas relacionadas con esta.

- Los alumnos de quinto año han de tener un conocimiento de Química más experto que los anteriores debido a su paso por la Universidad y, además, por estar a punto de integrarse a la vida laboral como profesionales.

Lo citado anteriormente convierte a ambos grupos en adecuados para valorar su grado de preparación frente a los desafíos diarios de las sociedades modernas y, así, obtener una idea bastante aproximada de las competencias que se han adquirido a lo largo de ambos periodos educativos.

Es decir, se elige a los alumnos de primer y quinto ańo para realizar el contraste, partiendo de la hipótesis de que las características de los de primero son equivalentes a las de los de quinto hace cinco años atrás.

\section{Estrategia de recogida de la información}

En este apartado se describe la estrategia de recogida de la información, para la que se han elaborado y utilizado dos cuestionarios: uno de contexto y otro de competencia cientifica.

- Cuestionario de contexto: mediante este instrumento se indaga si existen algunas características del contexto sociocultural de los estudiantes que pudieran influir en su rendimiento en ciencias. Se reconoce que existen múltiples factores de contexto más o menos relevantes, según diferentes criterios. Por lo tanto, en este trabajo, se ha elaborado un cuestionario de modo que permita obtener información acerca de los siguientes indicadores: la edad, el género (a través del nombre), la procedencia, el centro educativo o bachillerato del que proviene (modalidad con la que finalizó los estudios y si es público o privado) y el perfil educativo y la ocupación de los padres.

- Cuestionario de competencia científica: mediante este instrumento se explora la competencia cientifica del alumnado. Se ha tomado como base la definición de competencia cientifica del informe PISA 2006, que puede caracterizarse por cuatro aspectos interrelacionados (OCDE 2002, 2004 y 2006): capacidades, conocimientos, áreas de aplicación y actitudes; y se han seleccionado algunas pruebas de dicho informe. Consecuentemente, para la elaboración de dicho cuestionario se han adaptado dichas pruebas al nivel universitario y se han diseñado otras de autoría propia. En ellas se solicita al alumnado la escritura e interpretación del lenguaje simbólico de los conocimientos químicos y el uso de modelos de representación macro y microscópico (Johnstone, 1982); se indaga sobre cómo utilizan el concepto de ácido en dos temas distintos (lluvia ácida y caries), y se exploran determinadas capacidades y conocimientos del alumnado. En este trabajo no se aborda el estudio sobre actitudes.

Todos los alumnos tuvieron el mismo cuestionario sobre la mesa y, para responderlo, se estableció una hora de duración y no se necesitó más que papel y lápiz.

El cuestionario de competencia científica elaborado consistió en 17 preguntas, divididas en 2 temas, lluvia ácida (10) y caries (7). Teniendo en cuenta PISA 2006 (OCDE, 2006), estos temas fueron seleccionados debido a que las áreas de aplicación (medio ambiente y salud), las categorías del conocimiento de la ciencia y del conocimiento acerca de la ciencia (los sistemas físicos, los sistemas vivos, la investigación cientifica y las explicaciones científicas, respectivamente) están íntimamente relacionadas con las carreras de Bioquímica y Biotecnología, en las cuales se llevó a cabo este estudio. Cada una de las diecisiete cuestiones permite evaluar una de las subcompetencias (o capacidades) cientificas (ICC, EFC o UPC) y requiere el empleo de una o varias categorías de conocimiento. 
Cada tema (lluvia ácida y caries) comenzó con la presentación de un texto introductorio relacionado con una situación científica de la vida real (material de estímulo). A este texto le siguieron las preguntas o ítems que los alumnos y alumnas debían responder.

Estas se presentaron en diferentes formatos y se podían resolver mediante dos tipos de respuestas:

- Respuesta de elección múltiple: se debía seleccionar una sola opción entre varias alternativas dadas, normalmente cuatro. Esta podía ser simple (2 preguntas) o compleja (3 preguntas) si, además de elegir la opción correcta, correspondía explicar el fundamento de dicha elección.

- Respuesta de construcción: esta podía ser cerrada (6 preguntas) o abierta (6 preguntas).

Según PISA 2006 (OCDE, 2006), las preguntas se centran en situaciones relacionadas con el yo, la familia y los grupos de compańeros (personal), la comunidad (social) y la vida a escala mundial (global).

Por razones de espacio, solamente se reproducen tres ejemplos de las preguntas del tema lluvia ácida en los anexos 1, 2 y 3 . En estos, se pueden observar las preguntas propiamente dichas dirigidas a los alumnos en la parte A. Luego, para información del lector de este trabajo, se indican los criterios de corrección y las características de las preguntas que figuran con letra cursiva en la parte B. Por lo tanto, en los anexos puede observarse cada pregunta con el siguiente esquema: A. Información y pregunta para el alumno (introducción del tema y su planteamiento):

a. Tema. Texto introductorio del tema.

b. Planteamiento del problema a resolver.

B. Criterios de corrección (ver detalle en el siguiente apartado: «Estrategia de análisis de la información») y características de las preguntas:

a. Puntuación. Criterios de corrección.

b. Descripción de ciertas características incluyendo:

- Tipo de ejercicio o respuesta.

- Capacidad o competencia científica.

- Categoría de conocimiento.

- Área de aplicación.

- Marco.

- Comentario.

- Validez y fiabilidad

Para la validez interna en el diseño del cuestionario de competencia cientifica, se ha utilizado el método interno racional o de contenido, el cual permite determinar la validez de un instrumento, comprobando si mide aquello para lo que se ha elaborado. Es decir, en este caso, si mide las subcompetencias cientificas: ICC, EFC y UPC. De este modo, para llegar a la versión final del instrumento se solicitó a un grupo de expertos de nuestras cátedras que leyeran y consideraran los textos que se evalúan en cada ítem y sus correspondientes claves de corrección.

Entre los métodos más habituales para medir el grado de fiabilidad de los instrumentos se ha optado por el alfa de Cronbach. El valor de $\alpha=0,78$ indica que los resultados de opinión de los 122 alumnos, respeto a los 17 ítems considerados, se encuentran correlacionados de manera altamente fiable y muy aceptable.

\section{Estrategia de análisis de la información}

Las categorías "puntuación máxima», "puntuación parcial» y «sin puntuación» dividen las respuestas de los alumnos en tres grupos, según la capacidad que demuestren a la hora de responder a la pregunta formulada. Una respuesta calificada con una "puntuación máxima» no indica que la respuesta sea 
absolutamente correcta en términos científicos, pero sí que se posee el nivel adecuado de comprensión del tema para un alumno universitario científicamente competente. Las respuestas menos elaboradas, o con un menor grado de corrección, pueden obtener una "puntuación parcial», mientras que las preguntas cuyas respuestas son incorrectas, irrelevantes o que no se contestan, quedarán recogidas bajo la categoría «sin puntuación».

La puntuación posible de las preguntas construidas cerradas o de opción múltiple oscila entre 0 y 2 puntos. Las preguntas construidas abiertas que pueden tener distintos niveles de corrección tendrán diferentes puntuaciones entre 0,1 y 2 puntos. Sirvan de ejemplos los anexos 1, 2 y 3.

Debido a la dispersión numérica de las puntuaciones que se producen, los resultados se agrupan en niveles de rendimiento.

Según la OCDE 2006:

En la definición aquí propuesta, la competencia cientifica se concibe como un continuo que abarca desde los niveles de competencia científica más bajos hasta los más avanzados. Dicho de otra manera, se considera que las personas poseen diversos grados de competencia cientifica y no que posean o carezcan de competencia científica en términos absolutos (Bybee, 1997). Por ejemplo, un estudiante con un nivel de competencia menos desarrollado puede ser capaz de recordar conocimientos científicos sencillos y de emplear conocimientos científicos de uso corriente para sacar y evaluar conclusiones. En cambio, un alumno con un nivel de competencia cientifica más avanzado podrá crear y emplear modelos con objeto de hacer predicciones y dar explicaciones, analizar investigaciones científicas, relacionar entre sí datos que puedan constituirse en pruebas, evaluar explicaciones alternativas de un mismo fenómeno y exponer sus conclusiones con precisión (p. 26).

Para el análisis del Rendimiento total de competencia cientifica (RTCC), se consideró como línea de base o promedio el $50 \%$ del total de la puntuación, si todas las respuestas hubieran obtenido la puntuación máxima. Es decir, sobre un total de 17 preguntas, cuya máxima valoración es de 2 puntos cada una y suman 34 puntos (100\%), hemos fijado en 17 puntos $(50 \%)$ la calificación deseable para el nivel medio de rendimiento.

Para las diferentes subcompetencias se estableció como línea de base o promedio el 50\% del total de la puntuación de cada una de ellas, si todas las respuestas hubieran obtenido la puntuación máxima. Es decir, para:

- Identificar cuestiones cientificas (ICC), sobre un total de 3 preguntas, cuya máxima valoración es de 2 puntos cada una y en total suman 6 puntos (100\%), se fijó en 3 puntos (50\%) la calificación deseable para el nivel medio de rendimiento.

- Explicar fenómenos científicos (EFC), sobre un total de 10 preguntas, cuya máxima valoración es de 2 puntos cada una y suman en total 20 puntos (100\%), se fijó en 10 puntos (50\%) la calificación deseable para el nivel medio de rendimiento.

- Utilizar pruebas cientificas (UPC), sobre un total de 4 preguntas, cuya máxima valoración es de 2 puntos cada una y en total suman 8 puntos (100\%), se fijó en 4 puntos $(50 \%)$ la calificación deseable para el nivel medio de rendimiento.

Para comunicar los resultados mediante categorías, se llevó a cabo una adaptación al procedimiento de evaluación cualitativa y comunicación en porcentajes propuesto por Biggs (2005, p. 236). De acuerdo con este criterio, se presentan los niveles de competencia cientifica según los rangos de puntuaciones obtenidos, expresados en porcentajes, de la siguiente manera:

- Nivel máximo, entre 100 y 70\%.

- Nivel medio, de 70 a 50\%.

- Nivel bajo, menor que 50\%. 
Por ejemplo, un alumno que obtuvo las siguientes puntuaciones: $R T C C=13$ puntos, $I C C=5, E F C$ $=4$ y $U P C=4$, corresponde respectivamente a un 38\% del total de RTCC, un $83 \%$ de ICC, un $20 \%$ de $E F C$ y un $50 \%$ de $U P C$. En consecuencia, y según el criterio adoptado, este alumno pertenece a los niveles: bajo en $R T C C$, máximo para ICC, bajo en EFC y medio para UPC.

Posteriormente se contabilizó la cantidad de alumnos en cada categoría o nivel según lo descrito con anterioridad, y se calculó el respectivo porcentaje del total de estos para cada submuestra.

\section{RESULTADOS}

\section{Rendimiento total de competencia científica (RTCC) y su relación con ciertas variables del contexto}

Se han explorado ciertos antecedentes y circunstancias sociales y culturales de los alumnos que realizan la evaluación. Si bien existen otras variables, más o menos relevantes, los resultados que se exponen a continuación ayudan a describir algunas características de la muestra y su posible incidencia en el rendimiento de la competencia científica.

\section{Factores personales}

- Procedencia

Se ha considerado la distancia de cuarenta kilómetros como límite para diferenciar dos categorías: cercana y lejana. En la tabla 1, se recoge el porcentaje de alumnos según la distancia desde la que se desplaza el alumnado para asistir a clase y las notas medias de los resultados (expresadas en porcentaje, con un nivel de confianza del 95\%), según la procedencia.

Tabla 1 .

Porcentaje y RTCC del alumnado, por procedencia con respecto a la zona de la Universidad

\begin{tabular}{|c|c|c||c|c|}
\cline { 2 - 5 } \multicolumn{1}{c|}{} & \multicolumn{3}{c|}{ Procedencia } \\
\cline { 2 - 5 } \multicolumn{1}{c|}{} & \multicolumn{3}{c|}{ Cercana } & \multicolumn{2}{c|}{ Lejana } \\
\hline Año y carrera & $\%$ & RTCC Nota media (\%) & $\%$ & RTCC Nota media (\%) \\
\hline $1^{\circ} \mathrm{B}$ & 45 & 11 & 55 & 14 \\
\hline $1^{\circ} \mathrm{LB}$ & 38 & 11 & 62 & 11 \\
\hline $5^{\circ} \mathrm{B}$ & 33 & 21 & 67 & 19 \\
\hline $5^{\circ} \mathrm{LB}$ & 50 & 18 & 50 & 18 \\
\hline
\end{tabular}

Según la tabla 1 , se observa que en $1 .^{\circ} \mathrm{B}, 1 .^{\circ} \mathrm{LB}$ y $5 .^{\circ} \mathrm{B}$, la mayoría de los alumnos provienen de lugares alejados con respecto a la zona de la Universidad. Principalmente la procedencia lejana es del interior de las provincias de Santa Fe y de Entre Ríos. En el caso de 5. ${ }^{\circ}$ LB hay igual porcentaje de alumnos en ambas categorías.

Se puede observar que en $R T C C$ no se hallaron diferencias significativas por procedencia. Es decir, aunque el porcentaje de alumnos "cercanos" $\mathrm{y}$ "lejanos» en $1.0^{\circ} \mathrm{y}$ en $5 .{ }^{\circ}$ de ambas titulaciones presenta diferencias, esta característica de contexto del alumnado no ha influido en diferencias significativas en el rendimiento. 
- Edad-Años de estudio

Del análisis de las diferentes edades del alumnado, tanto en primero como en quinto año de la facultad, en ambas carreras los ingresantes tienen un promedio de entre 18 y 19 años, y en $5 .^{\circ}$ ańo las edades promedio están entre 22 y 24 años.

En la tabla 2 se recogen las notas medias de los resultados (expresadas en porcentaje, con un nivel de confianza del 95\%), según la edad-años de estudio.

Se toman dos rangos de edades, para los de 1.0: de 17 a 19 ańos y mayor e igual a 20. Para los de $5 .{ }^{\circ}$ : de 21 a 23 ańos y mayor e igual a 24.

Tabla 2.

Rendimiento total de competencia científica del alumnado, por edad

\begin{tabular}{|c|c|c|c|c|}
\cline { 2 - 5 } \multicolumn{1}{c|}{} & \multicolumn{4}{c|}{ Edad (años) } \\
\cline { 2 - 5 } \multicolumn{1}{c|}{} & $17-19$ & Igual o mayor a 20 & $21-13$ & Igual o mayor a 24 \\
\hline $\begin{array}{c}\text { Año y } \\
\text { carrera }\end{array}$ & $\begin{array}{c}R T C C \text { Nota media } \\
(\%)\end{array}$ & $\begin{array}{c}R T C C \text { Nota media } \\
(\%)\end{array}$ & $\begin{array}{c}R T C C \text { Nota media } \\
(\%)\end{array}$ & $\begin{array}{c}R T C C \text { Nota media } \\
(\%)\end{array}$ \\
\hline $1^{\circ} \mathrm{B}$ & 13 & 11 & - & - \\
\hline $1^{\circ} \mathrm{LB}$ & 11 & 10 & - & - \\
\hline $5^{\circ} \mathrm{B}$ & - & - & 19 & 20 \\
\hline $5^{\circ} \mathrm{LB}$ & - & - & 19 & 17 \\
\hline
\end{tabular}

Para ambos rangos, en $R T C C$, no se hallaron diferencias por edad entre los alumnos de $1 .{ }^{\text {er }}$ año, ni entre los de $5 .^{\circ}$ entre sí.

- Género

Otra de las variables personales exploradas fue el género de los estudiantes. En la tabla 3, se recogen los porcentajes de género, femenino $(\mathrm{F})$ y masculino $(\mathrm{M})$, tanto en primero como en quinto ańo de la facultad, y las notas medias de los resultados (expresadas en porcentaje, con un nivel de confianza del 95\%), según el género.

Tabla 3.

Porcentaje y $R T C C$ del alumnado, por género

\begin{tabular}{|c|c|c||c|c|}
\cline { 2 - 5 } \multicolumn{1}{c|}{} & \multicolumn{4}{c|}{ Género } \\
\cline { 2 - 5 } \multicolumn{1}{c|}{} & \multicolumn{3}{c|}{ M } \\
\hline Año y carrera & $\%$ & RTCC Nota media (\%) & $\%$ & $R T C C$ Nota media (\%) \\
\hline $1^{\circ} \mathrm{B}$ & 83 & 39 & 17 & 28 \\
\hline $1^{\circ} \mathrm{LB}$ & 70 & 33 & 30 & 29 \\
\hline $5^{\circ} \mathrm{B}$ & 73 & 55 & 27 & 77 \\
\hline $5^{\circ} \mathrm{LB}$ & 66 & 47 & 34 & 61 \\
\hline
\end{tabular}

Como se observa en la tabla 3, en ambas carreras, y en ambos años, el porcentaje de género femenino es mayor que el porcentaje masculino. 
En RTCC se hallaron diferencias por género: en $1 .^{\text {er }}$ año se puede observar (tabla 3) que el género femenino obtuvo mejores notas promedio y, en $5 .^{\circ}$ año, es el género masculino el que alcanzó medias más altas.

\section{Factores institucionales-gestión de escuela secundaria y modalidad}

Los contenidos comunes en la ciencia han de ser dominados por todos los estudiantes de la escuela secundaria superior tanto de gestión pública como privada, independientemente de la modalidad elegida, es decir, el bachillerato de procedencia.

El porcentaje de estudiantes según la gestión de estudios secundarios y los promedios de notas en $R T C C$ (expresadas en porcentaje, con un nivel de confianza del 95\%), por escuela pública o privada, se presentan en la tabla 4.

Tabla 4.

Porcentaje y RTCC del alumnado, por gestión de estudios secundarios

\begin{tabular}{|c|c|c||c|c|}
\cline { 2 - 4 } \multicolumn{1}{c|}{} & \multicolumn{4}{c|}{ Gestión de estudios secundarios } \\
\cline { 2 - 5 } \multicolumn{1}{c|}{} & \multicolumn{2}{c|}{ Pública } & \multicolumn{2}{c|}{ Privada } \\
\hline Año y carrera & $\%$ & $\begin{array}{c}R T C C \\
\text { Nota media } \\
(\%)\end{array}$ & $\begin{array}{c}\text { RTCC } \\
\text { Nota media } \\
(\%)\end{array}$ \\
\hline $1^{\mathbf{0}} \mathrm{B}$ & 60 & 37 & 40 & 36 \\
\hline $1^{\mathbf{0}} \mathrm{LB}$ & 53 & 35 & 47 & 30 \\
\hline $5^{\mathbf{0}} \mathrm{B}$ & 54 & 59 & 46 & 53 \\
\hline $5^{\mathbf{0}} \mathrm{LB}$ & 52 & 59 & & 50 \\
\hline
\end{tabular}

En las cuatro submuestras, se observa que más del $50 \%$ de los alumnos provienen de escuelas públicas. Según la tabla 4, se percibe que los estudiantes de las escuelas públicas obtuvieron un porcentaje de la nota promedio ligeramente mayor que los de las escuelas privadas.

Existen cinco modalidades para secundaria superior o polimodal: $(\mathrm{CN})$ Ciencias Naturales, $(\mathrm{CS})$ Ciencias Sociales, (EG) Economía y Gestión, (PBS) Producción de bienes y servicios, y (CAD) Comunicación, Arte y Diseño. La distribución de porcentaje de alumnos y los promedios de notas en RTCC (expresadas en porcentaje, con un nivel de confianza del 95\%), teniendo en cuenta la modalidad, se presentan en la tabla 5 .

Tabla 5 .

Porcentaje y RTCC del alumnado, por modalidad de estudios secundarios

\begin{tabular}{|c|c|c|c|c|c|c|c|c|}
\hline \multirow{3}{*}{ Año y carrera } & \multicolumn{8}{|c|}{ Modalidad de estudios secundarios } \\
\hline & \multicolumn{2}{|r|}{$\mathrm{CN}$} & \multicolumn{2}{|r|}{$\mathrm{CS}$} & \multicolumn{2}{|r|}{ EG } & \multicolumn{2}{|r|}{ PBS } \\
\hline & $\%$ & $\begin{array}{c}R T C C \\
\text { Nota media } \\
(\%) \\
\end{array}$ & $\%$ & $\begin{array}{c}R T C C \\
\text { Nota media } \\
(\%)\end{array}$ & $\%$ & $\begin{array}{c}R T C C \\
\text { Nota media } \\
(\%)\end{array}$ & $\%$ & $\begin{array}{c}R T C C \\
\text { Nota media } \\
(\%)\end{array}$ \\
\hline $1^{\circ} \mathrm{B}$ & 55 & 41 & 11 & 29 & 18 & 27 & 16 & 39 \\
\hline $1^{\circ} \mathrm{LB}$ & 37 & 36 & 9 & 20 & 21 & 36 & 33 & 38 \\
\hline $5^{\circ} \mathrm{B}$ & 45 & 59 & 22 & 53 & 22 & 56 & 11 & 74 \\
\hline $5^{\circ} \mathrm{LB}$ & 40 & 53 & 13 & 53 & 27 & 47 & 20 & 74 \\
\hline
\end{tabular}


Tras analizar los datos totales presentados en la tabla 5, se observa que la mayoría de los alumnos provienen de la modalidad en Ciencias Naturales. Seguidamente, prevalecen Producción de bienes y servicios (técnicos) y Economía y Gestión (contables).

Para las cinco modalidades de secundaria superior o polimodal -(CN) Ciencias Naturales, (CS) Ciencias Sociales, (EG) Economía y Gestión, (PBS, técnicos) Producción de bienes y servicios y (CAD) Comunicación, Arte y Diseño-, en la tabla 5 se puede observar que, respecto a la modalidad de estudios elegida, en general las mejores notas medias provienen de PBS. Además, en $1 .{ }^{\circ} \mathrm{B}$, las calificaciones son más altas en Ciencias Naturales $(\mathrm{CN})$ y en PBS que en CS y EG.

\section{Factores del entorno familiar}

A los efectos de presentar información sobre el contexto socioeconómico y cultural de los estudiantes y, en particular, de variables que pueden incidir con más fuerza en el desempeño educativo, se exponen a continuación los siguientes aspectos:

- La educación de los padres

En la tabla 6, se recogen los porcentajes de las respuestas de los alumnos sobre el nivel de educación de las madres $(\mathrm{M})$ y de los padres $(\mathrm{P})$ y las notas medias de RTCC (expresadas en porcentaje, con un nivel de confianza del 95\%), según dicho nivel.

Tabla 6.

Porcentaje y RTCC del alumnado, por nivel de estudios de los padres

\begin{tabular}{|c|c|c|c|c|c|c|c|c|c|c|c|c|c|c|c|c|}
\hline & \multicolumn{16}{|c|}{ Estudios } \\
\hline & \multicolumn{4}{|c|}{ Primarios } & \multicolumn{4}{|c|}{ Secundarios } & \multicolumn{4}{|c|}{ Terciarios } & \multicolumn{4}{|c|}{ Universitarios } \\
\hline & \multicolumn{2}{|c|}{$\mathbf{M}$} & \multicolumn{2}{|c|}{$\mathrm{P}$} & \multicolumn{2}{|c|}{ M } & \multicolumn{2}{|c|}{$\mathrm{P}$} & \multicolumn{2}{|c|}{ M } & \multicolumn{2}{|c|}{$\mathrm{P}$} & \multicolumn{2}{|c|}{ M } & \multicolumn{2}{|c|}{$\mathrm{P}$} \\
\hline $\begin{array}{l}\text { Año y } \\
\text { carrera }\end{array}$ & $\%$ & $\begin{array}{c}R T C C \\
\text { Nota } \\
\text { media } \\
(\%)\end{array}$ & $\%$ & $\begin{array}{c}R T C C \\
\text { Nota } \\
\text { media } \\
(\%)\end{array}$ & $\%$ & $\begin{array}{c}R T C C \\
\text { Nota } \\
\text { media } \\
(\%)\end{array}$ & $\%$ & $\begin{array}{c}R T C C \\
\text { Nota } \\
\text { media } \\
(\%)\end{array}$ & $\%$ & $\begin{array}{c}R T C C \\
\text { Nota } \\
\text { media } \\
(\%)\end{array}$ & $\%$ & $\begin{array}{c}R T C C \\
\text { Nota } \\
\text { media } \\
(\%)\end{array}$ & $\%$ & $\begin{array}{c}R T C C \\
\text { Nota } \\
\text { media } \\
(\%)\end{array}$ & $\%$ & $\begin{array}{c}R T C C \\
\text { Nota } \\
\text { media } \\
(\%)\end{array}$ \\
\hline $1^{\circ} \mathrm{B}$ & 13 & 13 & 24 & 13 & 42 & 10 & 43 & 12 & 28 & 16 & 20 & 13 & 17 & 12 & 13 & 11 \\
\hline $1^{\circ} \mathrm{LB}$ & 8 & 12 & 25 & 9 & 42 & 12 & 36 & 12 & 22 & 10 & 14 & 12 & 28 & 10 & 25 & 10 \\
\hline $5^{\circ} \mathrm{B}$ & 21 & 19 & 39 & 20 & 29 & 17 & 31 & 19 & 36 & 19 & 15 & 13 & 14 & 21 & 15 & 19 \\
\hline $5^{\circ} \mathrm{LB}$ & 14 & 17 & 24 & 20 & 23 & 16 & 29 & 15 & 18 & 19 & 18 & 19 & 45 & 18 & 29 & 17 \\
\hline
\end{tabular}

Según la tabla 6, los mayores porcentajes son:

- En $1 .^{\circ} \mathrm{B}$ y $1 .^{\circ} \mathrm{LB}$, tanto padres como madres tienen estudios secundarios.

- En 5..$^{\circ}$, las madres tienen estudios terciarios y los padres primarios.

- En 5. LB, las madres tienen estudios universitarios y los padres igual número en estudios secundarios y universitarios.

En RTCC no se hallaron diferencias por nivel de estudios de los padres. Se puede apreciar que un mayor nivel de estudio de los padres no se refleja en un porcentaje de nota media de RTCC más elevada. 
- Ocupación de los padres

En la tabla 7 se recogen las respuestas de los alumnos sobre la ocupación de las madres (M) y de los padres (P), y las notas medias de RTCC (expresadas en porcentaje, con un nivel de confianza del 95\%), según la ocupación. La clasificación desempleado incluye tanto a quienes no tienen empleo como a las amas de casa. La categoría de empleado comprende a quienes trabajan por cuenta ajena, e independiente a quienes trabajan por cuenta propia, incluyendo las profesiones liberales.

Tabla 7.

Porcentaje y RTCC del alumnado, por ocupación de los padres

\begin{tabular}{|c|c|c|c|c|c|c|c|c|c|c|c|c|c|c|c|c|}
\hline & \multicolumn{16}{|c|}{ Ocupación } \\
\hline & \multicolumn{4}{|c|}{ Desempleado } & \multicolumn{4}{|c|}{ Jubilado } & \multicolumn{4}{|c|}{ Empleado } & \multicolumn{4}{|c|}{ Independiente } \\
\hline & \multicolumn{2}{|r|}{ M } & \multicolumn{2}{|r|}{$\mathrm{P}$} & \multicolumn{2}{|r|}{ M } & \multicolumn{2}{|r|}{$P$} & \multicolumn{2}{|c|}{ M } & \multicolumn{2}{|r|}{$\mathrm{P}$} & \multicolumn{2}{|c|}{$\mathbf{M}$} & \multicolumn{2}{|c|}{$P$} \\
\hline $\begin{array}{l}\text { Año y } \\
\text { carrera }\end{array}$ & $\%$ & \begin{tabular}{|c}
$R T C C$ \\
Nota \\
media \\
$(\%)$
\end{tabular} & $\%$ & $\begin{array}{c}R T C C \\
\text { Nota } \\
\text { media } \\
(\%)\end{array}$ & $\%$ & \begin{tabular}{|c}
$R T C C$ \\
Nota \\
media \\
$(\%)$
\end{tabular} & $\%$ & $\begin{array}{c}R T C C \\
\text { Nota } \\
\text { media } \\
(\%)\end{array}$ & $\%$ & $\begin{array}{c}R T C C \\
\text { Nota } \\
\text { media } \\
(\%)\end{array}$ & $\%$ & $\begin{array}{c}\text { RTCC } \\
\text { Nota } \\
\text { media } \\
(\%)\end{array}$ & $\%$ & $\begin{array}{c}R T C C \\
\text { Nota } \\
\text { media } \\
(\%)\end{array}$ & $\%$ & $\begin{array}{c}R T C C \\
\text { Nota } \\
\text { media } \\
(\%)\end{array}$ \\
\hline $1^{\circ} \mathrm{B}$ & 36 & 12 & 2 & 12 & 2 & 17 & 2 & 9 & 45 & 13 & 58 & 12 & 17 & 12 & 38 & 13 \\
\hline $1^{\circ} \mathrm{LB}$ & 29 & 10 & 9 & 11 & 6 & 12 & 3 & 7 & 38 & 12 & 38 & 11 & 27 & 11 & 50 & 11 \\
\hline $5^{\circ} \mathrm{B}$ & 42 & 19 & - & - & 8 & 25 & 8 & 9 & 42 & 19 & 42 & 17 & 8 & 14 & 50 & 22 \\
\hline $5^{\circ} \mathrm{LB}$ & 23 & 17 & 14 & 23 & - & - & - & - & 50 & 19 & 38 & 16 & 27 & 15 & 48 & 17 \\
\hline
\end{tabular}

De acuerdo con la tabla 7 se detecta que la mayoría de los padres son empleados.

En RTCC no se hallaron diferencias por ocupación de los padres. Cabe mencionar que el porcentaje de padres jubilados es muy bajo, por lo que no se puede considerar como determinante la nota media en esta categoría.

\section{Resultados de competencia científica}

En la tabla 8 se exponen los resultados de los rendimientos obtenidos para cada una de las subcompetencias cientificas evaluadas, expresados en porcentaje de alumnos que alcanzaron los distintos niveles de desempeño. En dicha tabla se expresan las siguientes categorías taxonómicas antes descritas: RTCC, ICC, EFC y UPC. 
Tabla 8.

Resultados de competencia científica

\begin{tabular}{|c|c|c|c|c|c|}
\hline \multirow[t]{2}{*}{ Calificación } & \multicolumn{2}{|c|}{$\begin{array}{l}\text { \% alumnos } \\
\text { Bioquímica }\end{array}$} & \multicolumn{2}{|c|}{$\begin{array}{c}\text { \% alumnos } \\
\text { Biotecnología }\end{array}$} & \multirow[t]{2}{*}{ Competencia } \\
\hline & $1^{0}$ año & $5^{\circ}$ año & $\overline{1}^{\circ}$ año & $5^{\circ}$ año & \\
\hline \multirow{4}{*}{$\begin{array}{l}\text { Nivel máximo } \\
\text { (\% puntuación } \\
\text { alcanzada } \\
100 \text { a } 70)\end{array}$} & 0 & 13 & 0 & 9 & RTCC \\
\hline & 17 & 44 & 14 & 32 & $I C C$ \\
\hline & 0 & 0 & 0 & 5 & $E F C$ \\
\hline & 6 & 56 & 3 & 23 & $U P C$ \\
\hline \multirow{4}{*}{$\begin{array}{c}\text { Nivel medio } \\
\text { (\% puntuación } \\
\text { alcanzada } \\
70 \text { a } 50)\end{array}$} & 23 & 56 & 3 & 50 & $\overline{R T C C}$ \\
\hline & 43 & 25 & 35 & 32 & $I C C$ \\
\hline & 17 & 37 & 8 & 50 & $E F C$ \\
\hline & 64 & 44 & 65 & 54 & $U P C$ \\
\hline \multirow{3}{*}{$\begin{array}{c}\text { Nivel bajo } \\
\text { (\% puntuación } \\
\text { alcanzada }\end{array}$} & 77 & 31 & 97 & 41 & RTCC \\
\hline & 40 & 31 & 51 & 36 & $I C C$ \\
\hline & 83 & 63 & 92 & 45 & $E F C$ \\
\hline$<50)$ & 30 & 0 & 32 & 23 & $U P C$ \\
\hline
\end{tabular}

\section{Rendimiento total de competencia cientifica (RTCC)}

En la tabla 8, se pone de manifiesto que en los primeros años de Bioquímica y de Biotecnología se encontró, respectivamente, que un 77 y un $97 \%$ de los alumnos están por debajo del nivel básico fijado. Esto significa que para este nivel inicial la mayoría de los alumnos de estas carreras, aun habiendo aprobado el ingreso universitario, no poseen una base sólida de conocimientos y habilidades provenientes de la etapa escolar.

El alumnado de $5 .^{\circ}$ año de Bioquímica y de Biotecnología muestra un sensible aumento en los porcentajes del nivel más alto, aunque la mayoría se distribuyen entre los niveles medio (56-50\%) y bajo (31-41\%). Considerando que se trata de alumnos del último año de estudio de carreras universitarias, estos hallazgos son llamativamente muy bajos.

Si se consideran, por su parte, las variaciones de rendimiento en ciencias de los grupos analizados en relación con los años de estudio, los análisis revelan una asociación positiva entre los dos factores. Pero también sugieren que, aunque es un prerrequisito para una educación de calidad, la cantidad de años en sí misma no es suficiente para conseguir altos niveles de resultados.

\section{Identificar cuestiones cientificas (ICC)}

Se puede observar (tabla 8) que es la subcompetencia en la que se obtienen mejores resultados.

Los primeros años de Bioquímica y de Biotecnología tienen, respectivamente, un 40 y un 51\% de los alumnos por debajo del nivel básico fijado.

El alumnado de quinto año de Bioquímica y de Biotecnología muestra un sensible descenso en los porcentajes del nivel más bajo: 31 y $36 \%$, respectivamente. 


\section{Explicar fenómenos cientificamente (EFC)}

Es, probablemente, la subcompetencia más trabajada en las aulas, ya que se basa en la aplicación de conceptos científicos. Sin embargo, se hallan, en general, los resultados más bajos (tabla 8).

Los primeros años de Bioquímica y de Biotecnología tienen, respectivamente, un 83 y un $92 \%$ de los alumnos por debajo del nivel básico fijado. Ambos presentan $0 \%$ en el nivel alto, y en el caso de $1 .^{\circ}$ de Bioquímica (17\%) duplica a $1 .^{\circ}$ de Biotecnología (8\%) en el nivel medio.

En 5..$^{\circ}$ de Bioquímica se observa una mejoría en los valores, aunque ningún alumno alcanza el nivel máximo. No obstante, no es tan visible como la que se produce en $5 .^{\circ}$ de Biotecnología, en la que disminuye, respecto a $1 .^{\circ}$, el nivel más bajo en un (45\%), el medio aumenta (50\%) y el alto aparece con un $5 \%$, frente al $0 \%$ en primero.

\section{Utilizar pruebas cientificas (UPC)}

Los primeros años de Bioquímica y de Biotecnología tienen, respectivamente, un 30 y un 32\% de los alumnos por debajo del nivel básico fijado. Ambos presentan el porcentaje más alto en el nivel medio: 64 y $65 \%$, respectivamente.

En 5. ${ }^{\circ}$ de Bioquímica, se observa una marcada mejora en los valores, sin llegar a tener ningún alumno en el nivel mínimo. Sin embargo, en $5 .{ }^{\circ}$ de Biotecnología no se aprecia un progreso tan significativo.

\section{CONCLUSIONES}

De esta investigación se han obtenido resultados que permiten identificar la competencia cientifica con la que ingresan y egresan los estudiantes, así como las similitudes y diferencias entre ellas. Además, se ha podido inferir determinadas características curriculares que podrían orientar sobre las prioridades para una enseñanza de calidad que fomente el desarrollo de las subcompetencias investigadas, así como algunas influencias del contexto sociocultural de los alumnos en su rendimiento en ciencias.

Aunque este estudio es exploratorio - pues los resultados se han obtenido mediante pruebas de lápiz y papel, ya que no hemos investigado al alumnado cuando trabaja en el laboratorio- y debemos ser prudentes al hacer generalizaciones sobre la base de los resultados, el análisis de la información obtenida de los cuestionarios ha permitido redactar algunas conclusiones en función de los problemas de investigación planteados.

Respecto a los dos primeros:

- ¿Cuál es el grado de competencia científica que poseen los alumnos de primero y último año de las carreras de Bioquímica y Biotecnología?

- ¿Cuáles son las similitudes y diferencias entre la competencia científica de los estudiantes de $1 .{ }^{\text {er }}$ y $5 .^{\circ}$ año en ambas orientaciones universitarias?

Respecto al rendimiento total en los primeros años de ambas carreras, se observan valores bajos. Luego de la instrucción, en los últimos años, dichos valores aumentan y de manera más apreciable en Bioquímica.

La subcompetencia identificar cuestiones cientificas (centrada en aspectos de la metodología de la ciencia) es la que mejores resultados presenta. Esto resulta sorprendente por la dificultad que entraña, ya que exige distinguir entre las cuestiones y los contenidos científicos y otros aspectos ya mencionados. 
Bioquímica alcanza los mejores resultados en las distintas etapas de estudio. Luego de los años de instrucción se observa que $5 .^{\circ} \mathrm{B}$ logra un mayor desarrollo de esta competencia que 5. ${ }^{\circ} \mathrm{LB}$.

La subcompetencia explicar fenómenos cientificamente (centrada en la aplicación de conceptos científicos) presenta los resultados más bajos en los cuatro grupos estudiados. $1 .^{\circ} \mathrm{B}$ comienza con mejores resultados que $1 .^{\circ} \mathrm{LB}$ y mejora con la instrucción, sin embargo parece ser que la instrucción en Biotecnología desarrolla y mejora esta capacidad en mayor grado, ya que $5 .^{\circ} \mathrm{LB}$ obtiene mejores puntuaciones que $5 .{ }^{\circ} \mathrm{B}$.

En ambas carreras, y en primer año, la subcompetencia utilizar pruebas científicas (centrada en las evidencias y la argumentación) posee valores semejantes. Posteriormente, los resultados mejoran con la instrucción. Este es un dato positivo, ya que utilizar pruebas científicas conlleva la capacidad de acceder a la información científica, así como la elaboración de argumentaciones y conclusiones basadas en pruebas científicas (Osborne, Erduran, Simon y Monk, 2001). Es la competencia en la cual se presentan las mayores diferencias entre los años de estudio de ambas carreras. Parece que la instrucción en Bioquímica desarrolla y mejora esta capacidad en mayor grado que la de Biotecnología.

\section{Respecto al tercero de los problemas:}

- ¿Ciertas características del contexto sociocultural de los alumnos influyen en el rendimiento de la competencia científica?

Sin pretender establecer conclusiones definitivas, pueden señalarse las siguientes aproximaciones para comprender los resultados expuestos:

- Si se observan las tablas 1 y 2 , el rendimiento total de competencia científica del alumnado, por procedencia y por edad, no revela diferencias significativas.

- Según la tabla 3, en el rendimiento total del alumnado por género, el género femenino obtiene un rendimiento levemente superior que el masculino en primer año y ligeramente inferior en quinto de ambas carreras.

- Las condiciones del entorno familiar, ya sea el nivel educativo y/o la ocupación de los padres, no tienen influencia en el rendimiento del alumnado, como ya se advirtió en las tablas 6 y 7.

- También se observa que la escuela y la instrucción influyen obviamente en la adquisición de conocimientos. En este sentido, la cantidad de horas de clases de ciencias (PBS y CN) y el carácter público de estas son aspectos que contribuyen a un mayor logro académico de los alumnos (tablas 4 y 5).

A raíz de este trabajo, se ha de meditar sobre la problemática que supone el desarrollo de las competencias específicas o profesionales de las carreras analizadas. En este sentido, parece necesario cambiar las estrategias y producir una transformación en el sistema de enseñanza y aprendizaje. Esto no implica que todas las actividades que se realizan en la enseñanza tradicional, tales como clases magistrales, exposiciones, prácticas de laboratorio, talleres, seminarios, etc., sean eliminadas, pero se requiere una formación didáctica de los docentes que los capacite para mejorar sus propias prácticas. Sin lugar a dudas, estos cambios demandarán mayor tiempo y trabajo y, eventualmente, contar con un equipo de apoyo. Por ello, es importante que las instituciones tengan una política de enseñanza-aprendizajeevaluación, infraestructuras y recursos humanos que propicien el cambio, cuya metodología dependerá además de otros factores y de las características de cada institución.

Latinoamérica muestra por ahora un panorama sombrío. Argentina, por ejemplo, tiene más del $50 \%$ de los alumnos por debajo del nivel básico de alfabetización científica en las pruebas de PISA 2006 (Gutiérrez, 2008; DINIECE-MEN, n.d.). Según lo expresa Gutiérrez (2008), con relación a los niveles de competencias, este país se encuentra en el nivel 1, por debajo del nivel mínimo para una al- 
fabetización científica básica (nivel 2). En el reciente estudio PISA 2009 (OECD, 2010), los resultados no han mejorado, pues Argentina ha ocupado el puesto 56..$^{\circ}$ de 64 países evaluados, respecto al 51. ${ }^{\circ}$ que ocupaba en 2006 entre 56 países de todo el mundo.

Para apropiarse del concepto de competencia y ser capaces de ayudar a los alumnos a desarrollar las suyas, los profesores han de pensar globalmente $y$, sobre todo, tener presente que los alumnos deben transferir lo aprendido a la toma de decisiones en relación con situaciones no trabajadas en clase explícitamente.

La definición de los grados y tipos de competencia permite facilitar el diagnóstico de los aspectos que necesitan atención en las carreras analizadas. Del análisis realizado y debido a las características de las carreras universitarias aquí estudiadas, además de lo que ya se hace bien en estos momentos, una de las implicaciones de este estudio podría ser mejorar el rendimiento de las subcompetencias citadas si en la instrucción se priorizan los siguientes aspectos:

- Identificar cuestiones científicas relevantes por medio de pruebas o procedimientos científicos.

- Insistir en las explicaciones científicas cualitativas.

- Trabajar la tipología textual científica y la simbólica.

Además, se plantean nuevos interrogantes que podrían dar lugar a futuras investigaciones en pos de mejorar la didáctica de ciertos temas relevantes para los perfiles profesionales de los bioquímicos y biotecnólogos.

Atendiendo a las dificultades descubiertas podemos pensar en otras nuevas líneas de trabajo:

- Realizar un estudio longitudinal de los alumnos a lo largo de ambas carreras.

- Diseñar y evaluar estrategias educativas y propuestas de enseñanza que puedan ayudar a los alumnos a mejorar su competencia en ciencias y a los profesores sus prácticas docentes.

- Estudiar los niveles epistémicos en las argumentaciones cuando los alumnos responden a preguntas abiertas.

- Validar la prueba mediante métodos externos (evaluación cíclica) facilitaría el seguimiento de los resultados de las innovaciones adoptadas.

- Establecer perfiles específicos para la resolución de problemas. Estos perfiles se pueden utilizar para crear nuevos tipos de tareas con distinto grado de dificultad y comparar los resultados (Stawitz, Rumann, Fleischer y Wirth, 2010).

- Buscar herramientas científicas para poder concluir, con la mayor precisión posible, qué es lo que está fallando cuando los resultados son bajos y qué se está haciendo bien cuando los resultados de los exámenes mejoran.

- Estudiar cualitativamente y en profundidad cada una de las respuestas.

Ante los resultados obtenidos en el presente trabajo, y a partir de los resultados que se pudiesen obtener desde las nuevas líneas de investigación antes propuestas, se podría reflexionar con fundamento sobre cuáles son los perfiles que se desean para los estudiantes que egresan de la educación secundaria y para los futuros graduados universitarios. En definitiva, sobre las perspectivas de la educación en América Latina (Tuning Proyect, 2007).

\section{AGRADECIMIENTOS}

A los proyectos EDU2012-38022-C02-01, del Ministerio de Economía y Competitividad de España, y CAI+D 2009-12/B030, de la Universidad Nacional del Litoral de Argentina. 


\section{REFERENCIAS BIBLIOGRÁFICAS}

Biggs, J. (2005). Calidad del aprendizaje universitario. Madrid: Narcea.

Bybee, R. W. (1997). Achieving scientific literacy: from purposes to practices. Portsmouth NH: Heinemann.

Cañas, A.; Martín-Díaz, M. J. y Nieda, J. (2007). Competencia en el conocimiento y la interacción con el mundo físico. La competencia cientifica. Madrid: Alianza Editorial.

Chamizo, J. A. y Izquierdo, M. (2007). Evaluación de las competencias de pensamiento científico. Alambique, 51, pp. 9-19.

Colás Bravo, M. P. y Buendía Eisman, L. (1998). Investigación educativa, 3. a ed. Sevilla: Alfar.

De Pro Bueno, A. (2007). De la enseñanza de los conocimientos a la enseñanza de las competencias. Alambique, 53, pp. 10-21.

DINIECE-MEN (n.d.). Informe Nacional PISA 2006. Programa Internacional para la Evaluación de los Estudiantes. Disponible en línea: <http://diniece.me.gov.ar/images/stories/diniece/evaluacion_educativa/internacionales/Inf-ReultadoPISA-2006.pdf> (Última consulta, 17 de marzo de 2009).

Domenech, V. (2003). El proyecto PISA. Un proyecto internacional para la búsqueda de indicadores de rendimiento. Alambique, 37, pp. 19-32.

Gutiérrez, S. (2008). La evaluación de las competencias científicas en PISA: perfiles en los estudiantes iberoamericanos. Alambique, 57, pp. 23-31.

IzQuierdo Aymerich, M. (2004). Un nuevo enfoque de la enseñanza de la química: contextualizar y modelizar. The Journal of the Argentine Chemical Society, 92 (4-6), pp. 115-136.

Jiménez Aleixandre, M. P. (2000). Modelos didácticos. En F. J. Perales Palacios y P. Cañal de León. Didáctica de las Ciencias Experimentales. Teoría y Práctica de la Enseñanza de las Ciencias. España: Marfil, pp. 165-186.

Jiménez Aleixandre, M. P. (2010). 10 ideas clave. Competencias en argumentación y uso de pruebas. Barcelona: Graó.

Johnstone, A. H. (1982). Macro and microchemistry. School Science Review, 64, pp. 377-379.

Labate, H. (2007). Science education: a (pending) chapter in the curriculum transformation in Argentina. Prospects, 37 (4), pp. 469-488. DOI 10.1007/s11125-008-9045-2.

http://dx.doi.org/10.1007/s11125-008-9045-2

Marco Stiefel, B. (2008). Competencias Básicas. Hacia un nuevo paradigma educativo. Madrid: NARCEA-MEPSYD.

Monereo Font, C. y Pozo Municio, J. I. (2001). ¿En qué siglo vive la escuela? El reto de la nueva cultura educativa. Cuadernos de Pedagogía, 298, pp. 50-55.

Monereo, C. y Pozo, J. I. (2007). Competencias para (con)vivir con el siglo Xxi. Cuadernos De Pedagogía, 370, pp. 12-18.

Monereo, C. (coord.); Álunarez, I. M.; Canal, M.; Castelló, M.; Cerrato, P.; Corcelles, M.; Duran, D.; Gómez, I.; Lemus, R.; Núñez, M.; Serrano, S. y Vicente, L. (2009). PISA COMO EXCUSA. Repensar la evaluación para cambiar la enseñanza. Barcelona: Graó.

OCDE (2002). Muestra de reactivos empleados en la evaluación PISA 2000. Aptitudes Para Lectura, Matemáticas y Ciencias. México: Editorial Santillana. S.A.

OCDE (2004). Marcos teóricos de PISA 2003: la medida de los conocimientos y destrezas en matemáticas, lectura, ciencias y resolución de problemas. Madrid: MEC e INECSE.

OCDE (2006). PISA 2006: Marco de la evaluación. Conocimientos y habilidades en Ciencias, Matemáticas y Lectura. Disponible en línea: <http://browse.oecdbookshop.org/oecd/pdfs/free/9806034e. pdf> (Última consulta, 20 de febrero de 2009). 
OCDE (2008). Informe PISA 2006. Competencias cientificas para el mundo del mañana. Madrid: Santillana Educación, S. L. http://dx.doi.org/10.1787/9789264040151-en

OCDE (2009). PISA 2009 Assessment Framework-Key Competencies in Reading, Mathematics and Science. Disponible en línea: <http://www.oecd.org/pisa/pisaproducts/44455820.pdf> (Última consulta, 10 de diciembre de 2010).

OCDE (2010). PISA 2009 Results: What Students Know and Can Do - Student Performance in Reading, Mathematics and Science (Volume I). Disponible en línea: <http://www.oecd.org/dataoecd/54/12/46643496.pdf> (Última consulta, 10 de diciembre de 2010).

OCDE (n. d.). El programa PISA de la OCDE. Qué es y para qué sirve. Disponible en línea: <http:// www.oecd.org/dataoecd/58/51/39730818.pdf> (Última consulta, 10 de febrero de 2009).

Osborne, J.; Erduran, S.; Simon, S. y Mon, M. (2001). Enhancing the Quality of Argumentation in School Science. School Science Review, 82 (301), pp. 63-70.

Perrenoud, P. (2004b). Diez nuevas competencias para enseñar. Barcelona: Graó.

Sнön, D. A. (1992). La formación de profesionales reflexivos. Hacia un nuevo diseño de la enseñanza y el aprendizaje en las profesiones. Barcelona: Paidós.

Stawitz, H.; Rumann, S.; Fleischer, J. y Wirth, J. (2010). Effects of task profiles on students performance in Pisa 2003. En M. F. Taşar y G. Çakmakcı (eds.). Contemporary science education research: international perspectives. Ankara, Turkey: Pegem Akademi, pp. 453-457. Disponible en línea: <http://lsg.ucy.ac.cy/esera/Book3.pdf> (Última consulta, 10 de enero de 2011).

Tuning Project (2007). Informe Final del Proyecto Tuning América Latina: Reflexiones yperspectivas de la Educación Superior en América Latina. Disponible en línea: <http://tuning.unideusto.org/tuningal/ index.php?option=com_docman\&Itemid=191\&task=view_category\&catid=22\&order $=$ dmdate published\&ascdesc=DESC $>$ (Última consulta, 14 de mayo de 2010).

Zabala, A. y Arnau, L. (2007). 11 ideas clave. Cómo aprender y enseñar competencias. Barcelona: Graó. 


\section{ANEXO 1 (ICC)}

\section{A.Información y pregunta para el alumno (introducción del tema y su planteamiento)} Pregunta 3: LLUVIA ÁCIDA

Diseña un experimento científico para comprobar que la lluvia ácida corroe el mármol. . . . . . . . . B. Criterios de corrección y características de las preguntas

LLUVIA ÁCIDA: puntuación de la pregunta 3

\section{Máxima puntuación: 2 puntos}

La respuesta debe contener una descripción del método utilizado en el que se identifiquen el problema objeto de investigación, las variables que intervienen (lluvia ácida/mármol) y los efectos o reacciones que se producen (relación entre variables).

El diseño experimental es netamente explicativo, por cuanto su propósito es demostrar que los cambios en la variable dependiente (mármol) fueron causados por la variable independiente (agua ácida).

Puntuación parcial: 1 punto

La respuesta incluye una descripción de condiciones, pero no establece variables ni una relación causa-efecto.

Ninguna puntuación: Otras respuestas. Sin respuesta.

Tipo de ejercicio: Respuesta construida abierta.

Capacidad: Identificar cuestiones científicas (ICC).

Categoría de conocimiento: Investigación científica. (Conocimiento acerca de la ciencia). Sistemas físicos. (Conocimiento de la ciencia).

Área de aplicación: Medio ambiente.

Marco o Contexto: Personal.

Comentario: Se pretende que el alumno elabore un diseño experimental netamente cualitativo, por cuanto la respuesta debe contener la descripción del método utilizado que permita demostrar que los cambios en la variable dependiente (mármol) son causados por la variable independiente (agua ácida). Es decir, se pretende que establezca con precisión una relación causa-efecto.

\footnotetext{
ANEXO 2 (EFC)

A. Información y pregunta para el alumno (introducción del tema y su planteamiento) Pregunta 9: LLUVIA ÁCIDA

a) ¿Cuál sería la ecuación química que simboliza la reacción entre el mármol (carbonato de calcio) y la lluvia ácida?

b) ¿Cómo se llama en la vida cotidiana el producto final de la misma?

B. Criterios de corrección y características de las preguntas

LLUVIA ÁCIDA: puntuación de la pregunta 9

Máxima puntuación: 2 puntos.

a) $\mathrm{CaCO}_{3(\mathrm{~s})}+\mathrm{H}^{+}{ }_{(\mathrm{aq})} \rightleftarrows \mathrm{Ca}^{2+}{ }_{(\mathrm{aq})}+\mathrm{HCO}_{3}^{-}{ }_{(\mathrm{aq})}$

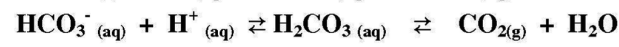

b) Yeso: $\mathrm{CaSO}_{4} \cdot 2 \mathrm{H}_{2} \mathrm{O}$

Puntuación parcial: 1 punto.

La respuesta incluye uno solo de los incisos.

Ninguna puntuacion: Otras respuestas. Sin respuesta.

Tipo de ejercicio: Respuesta construida cerrada.

Capacidad: Explicar fenómenos científicamente (EFC).

Categoría de conocimiento: Explicaciones científicas. (Conocimiento acerca de la ciencia). Sistemas físicos. (Conocimiento de la ciencia).

Área de aplicación: Medio ambiente.

Marco o Contexto: Personal.

Comentario: Se solicita la aplicación del lenguaje simbólico (nomenclatura química) de los conocimientos químicos y la utilización de modelo macroscópico.
} 


\section{ANEXO 3 (UPC)}

A. Información y pregunta para el alumno (introducción del tema y su planteamiento) Pregunta 4: LLUVIA ÁCIDA

A pesar de las actividades económicas e industriales de Barranquilla (Colombia), las cuales aumentan la probabilidad de acidificación de la atmósfera, esta ciudad se encuentra influenciada por la brisa del Mar Caribe, efecto que puede neutralizar cualquier proceso de acidificación en el medio. Este fenómeno se debe específicamente a la evaporación del agua de mar la cual, al reaccionar en la atmósfera con las sustancias productoras de lluvia ácida, ejerce una función neutralizadora.

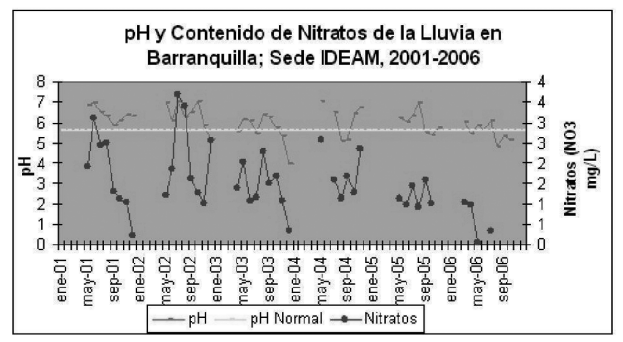

Gráfica: tomada de IDEAM

(http://www.ideam.gov.co:8080/sectores/Copia\%20de\%20Lluvia/LLuviaAcidaEIPrograma.html 05-03-10) La línea amarilla representa el $\mathrm{pH}$ normal de la lluvia $(5,65)$

¿Cuál de las siguientes afirmaciones se ve corroborada por los datos que figuran en el gráfico? Marca con una $\mathrm{X}$ la/s opción/es correcta/s.

$\square$ a. Los valores de $\mathrm{pH}$, generalmente por encima del rango normal, indican que no ha existido acidificación de la lluvia, situación generada muy probablemente por el efecto que la brisa marina ejerce sobre la ciudad.

$\square$ b. Los valores de $\mathrm{pH}$ mantienen un comportamiento con una preponderancia de valores por encima de 5.6, demostrando una notable disminución generalmente entre septiembre y noviembre de todos los años.

$\square$ c. Existe relación directa entre la concentración de nitratos y el pH en el año 2002.

$\square$ d. Los valores de $\mathrm{pH}$ mantienen un comportamiento de valores por debajo de 5.6, con disminución en enero de 2002 y el mismo mes de 2005.

\section{B. Criterios de corrección y características de las preguntas}

\section{LLUVIA ÁCIDA: puntuación de la pregunta 4}

\section{Máxima puntuación: 2 puntos}

Solamente la primera afirmación se ve corroborada por los datos que figuran en el gráfico.

Sin puntuación: Otras respuestas. Sin respuesta.

Tipo de ejercicio: Elección múltiple.

Capacidad: Utilización de pruebas cientificas (UPC).

Categoria de conocimiento: Explicaciones cientificas (Conocimiento acerca de la ciencia). Sistemas fisicos. (Conocimiento de la ciencia).

Área de aplicación: Medio ambiente.

Marco: Personal.

Comentario: Los datos (pruebas) se suministran en forma de gráfico. Para interpretar correctamente el gráfico hay que comprender de forma clara cuáles son las variables representadas en el mismo. 


\title{
Science competency of students enrolling and graduating at University
}

\author{
C. B. Falicoff, H. S. Odetti \\ Departamento de Química General e Inorgánica, Facultad de Bioquímica y Ciencias Biológicas, UNL. \\ Ciudad Universitaria Paraje El Pozo. CC 242. (3000), Santa Fe, Argentina. \\ falicoff@fbcb.unl.edu.ar, hodetti@fbcb.unl.edu.ar \\ J. M. Domínguez Castiñeiras \\ Departamento de Didáctica das Ciencias Experimentais, Facultade de Ciencias da Educación, USC. \\ Avda. Xoan XXIII, s/n. I 5704. Santiago de Compostela, España. \\ josemanuel.dominguez@usc.es
}

The aim of this study is to assess the science competency of students enrolling at the School of Biochemistry and Biological Sciences (first year of the courses of Biochemistry and Biotechnology of the Universidad Nacional del Litoral de Santa Fe, Argentina) and what competency they have when they finish University (fifth year of the same courses).

The concept of science competency has been drawn from the Program for International Student Assessment (PISA) 2006.

Pursuant to the above, the following questions are raised:

- Which science competency levels do students have in first and last-years of Biochemistry and Biotechnology courses?

- What are the similarities and differences between the students' science competency in first and fifth year in both university courses?

Is students' science competency performance influenced by certain features of the sociocultural context?

The study was conducted during the year 2010. Participating students were volunteers.

The research was developed with a sample of 84 first-year undergraduate students and 38 fifth-year students and the make-up was as follows:

- 47 students of 1 st year of Biochemistry.

- 37 students of 1 st year of Biotechnology.

- 16 students of 5 th year of Biochemistry.

- 22 students of 5 th year of Biotechnology.

The selection was performed using stratified sampling, seeking homogeneity within each stratum and heterogeneity among strata.

To collect the information, two ad hoc questionnaires were drafted: one with questions addressing three science subcompetencies (identifying scientific issues, explaining phenomena scientifically and using scientific evidence), the other one was used to examine the influence that certain contextual factors might have had on these competencies performance. The first questionnaire contained 17 questions and was divided into two topics: acid rain (10) and caries (7). Three levels of description and interpretation -macroscopic, microscopic and symbolic- of chemistry knowledge were assessed in different contexts, considered to be desirable from the viewpoint of academic knowledge.

In addition, analysis instruments were designed that enabled to process and categorize the information obtained.

The results indicate that first-year students start with a low performance level for all the science subcompetencies assessed. Identifying scientific issues is the subcompetency that shows the greatest results and explaining phenomena scientifically reveals the lowest ones in both courses.

After instruction, in the last years, the competencies develop favorably, more so among students of Biochemistry than among those of Biotechnology.

Certain features of the sociocultural context reveal no significant differences in the performance of science competency of students.

The results achieved in this study have led to new questions that will be addressed in future research. 